\title{
ON THE WEAK UNIFORM CONVEXITY OF $Q(R)$
}

\author{
SHEN YU-LIANG
}

(Communicated by Albert Baernstein II)

\begin{abstract}
We will discuss the geometry of the unit sphere in the Banach space of integrable holomorphic quadratic differentials on a Riemann surface and answer some questions posed by L.R. Goldberg (Proc. Amer. Math. Soc. 118 (1993), 1179-1185).
\end{abstract}

For a given hyperbolic Riemann surface $R$, that is, covered by the unit disk $\Delta$, we denote by $Q(R)$ the Banach space of all holomophic quadratic differentials $\phi(z) d z^{2}$ on $R$ with the $L^{1}$ norm

$$
\|\phi\|=\iint_{R}|\phi(z)| d x d y<\infty .
$$

It is well known that $Q(R)$ is of finite dimension if and only if $R$ is of finite type. Let $S(R)=\{\phi \in Q(R):\|\phi\|=1\}$ denote the unit sphere in $Q(R)$. We are going to study the geometry of $S(R)$, answering some questions of Goldberg [5].

In general, if $X$ is a Banach space, then $X^{*}$ is the Banach space of bounded real-linear functionals $l: X \rightarrow \mathbf{R}$. Now define $X$ to be smooth if for every unit vector $x$ in $X$ there is a unique $l$ in $X^{*}$ with $l(x)=\|l\|=1$. Note that our definition of smoothness is taken from Diestel [1] and differs from the definition in Goldberg [5]; however, Diestel proved that the two definitions are equivalent.

It is well known that $Q(R)$ is smooth (see [2] or [4]). If $\psi \in S(R)$, the unique functional $\psi_{*}$ in $Q(R)^{*}$ with $\psi_{*}(\psi)=\left\|\psi_{*}\right\|=1$ is

$$
\psi_{*}(\phi)=\operatorname{Re} \iint_{R} \phi(z) \frac{\overline{\psi(z)}}{|\psi(z)|} d x d y, \quad \phi \in Q(R) .
$$

It is also well known that $Q(R)$ is strictly convex, meaning (as usual) that every point of $S(R)$ is an extreme point of the closed unit ball.

Received by the editors July 26, 1994 and, in revised form, December 22, 1994.

1991 Mathematics Subject Classification. Primary 30F30, 30C70, 30F60.

Key words and phrases. Quadratic differential, weak uniform convexity, Hamilton sequence.

The author was supported in part by Jiangsu Provincial Natural Science Foundation. 
A Banach space $X$ is said to be uniformly convex if for all sequences $\left(x_{n}\right)$ and $\left(y_{n}\right)$ in the closed unit ball, $\left\|x_{n}+y_{n}\right\| \rightarrow 2$ implies $\left\|x_{n}-y_{n}\right\| \rightarrow 0$. It is obvious that a finite dimensional strictly convex Banach space is uniformly convex (in particular $Q(R)$ is uniformly convex if $R$ has finite type), but the infinite dimensional case is more complicated. In her study of the convexity of $Q(\Delta)$, Goldberg uses two weaker notions.

Following Goldberg [5] we say that $X$ is uniformly convex at the unit vector $x$ if for all sequences $\left(x_{n}\right)$ in the closed unit ball, $\left\|x_{n}+x\right\| \rightarrow 2$ implies $x_{n} \rightarrow x$. If $X$ is smooth, $x$ is a unit vector, and $x_{*}$ is the unique functional of norm one such that $x_{*}(x)=1$, we say that $X$ is weakly uniformly convex at $x$ if for all sequences $\left(x_{n}\right)$ in the unit sphere, $x_{*}\left(x_{n}\right) \rightarrow 1$ implies $x_{n} \rightarrow x$.

If $x_{*}\left(x_{n}\right) \rightarrow 1$, then $x_{*}\left(x_{n}+x\right) \rightarrow 2$, so $\left\|x_{n}+x\right\| \rightarrow 2$. Therefore uniform convexity at $x$ implies weak uniform convexity at $x$. Uniform convexity trivially implies uniform convexity at each unit vector $x$, so if $R$ is a finite Riemann surface, then $Q(R)$ is weakly uniformly convex at every point of $S(R)$. That solves Problem 3 of Goldberg [5].

To study the weak uniform convexity of $Q(R)$ in the infinite dimensional case we use Hamilton sequences. Let $l$ be a linear functional on $Q(R)$. By definition, a Hamilton sequence for $l$ is a sequence $\left(\phi_{n}\right)$ in $S(R)$ such that $l\left(\phi_{n}\right) \rightarrow\|l\|$. The Hamilton sequence $\left(\phi_{n}\right)$ is called degenerate if $\phi_{n} \rightarrow 0$ locally uniformly in $R$.

Now we can state our main result.

Theorem 1. The Banach space $Q(R)$ is weakly uniformly convex at $\psi \in S(R)$ if and only if the linear functional $\psi_{*}$ does not possess any degenerate Hamilton sequence.

Remark. Theorem 1 gives a characterization of the flat points in $S(R)$ where $Q(R)$ is not weakly uniformly convex; this gives an answer to Goldberg's Problem 2. A solution to her Problem 1 will be given in $\S 3$.

The paper is organized as follows: We prove Theorem 1 in $\S 2$. In $\S 3$, we obtain some consequences by using Strebel's Frame Mapping Condition from the theorey of quasiconformal mappings. Finally, in $\S 4$, we discuss the uniform convexity of the Banach space $Q(R)$.

We first point out that Hamilton sequences obey a well known principle which is implict in Strebel [7] and also Harrington-Ortel [6]. The principle is this: If $l$ is a nontrivial linear functional on $Q(R)$, then either $l$ has a degenerating Hamilton sequence or else every Hamilton sequence converges (in norm) to the unique $\phi$ in $S(R)$ such that $l(\phi)=\|l\|$. These two possibilities are obviously mutually exclusive.

Now we give the proof of Theorem 1 .

Assume first that $Q(R)$ is weakly uniformly convex at $\psi \in S(R)$. Let $\left(\phi_{n}\right)$ be any Hamilton sequence for $\psi_{*}$. Since $\psi_{*}\left(\phi_{n}\right) \rightarrow 1$, the weak uniform convexity implies that $\phi_{n} \rightarrow \psi$, so every Hamilton sequence for $\psi_{*}$ converges in norm to $\psi$, and no Hamilton sequence for $\psi_{*}$ is degenerate.

Conversely, suppose $\psi \in S(R)$ and no Hamilton sequence for $\psi_{*}$ is degenerate. We shall prove that $Q(R)$ is weakly uniformly convex at $\psi$. Let $\left(\phi_{n}\right)$ be a sequence in $S(R)$ such that $\psi_{*}\left(\phi_{n}\right) \rightarrow 1$. Since $\left(\phi_{n}\right)$ is a Hamilton sequence for $\psi_{*}$, the above-stated principle implies that $\phi_{n} \rightarrow \psi$ as required. 
For an extremal quasiconformal mapping $f: R \rightarrow R^{\prime}$, we denote by $H(f)$ the boundary dilatation of $f$ (see Strebel [8] or Gardiner $[4, \S 6.8]$ for the definition) and by $K_{0}(f)$ its maximal dilatation. For a given $\psi \in S(R)$, choose $k$ in the open interval $(0,1)$ and denote by $f_{\psi}$ the extremal quasiconformal mapping on $R$ whose Beltrami coefficient is $k \bar{\psi} /|\psi|$.

Theorem 2. If $\psi \in S(R)$, then $Q(R)$ is weakly uniformly convex at $\psi$ if and only if $H\left(f_{\psi}\right)<K_{0}\left(f_{\psi}\right)$.

Proof. By Strebel's Frame Mapping Condition (see [4] or [8]) and a recent result of Earle-Li [3], if $\psi \in S(R)$, then $H\left(f_{\psi}\right)<K_{0}\left(f_{\psi}\right)$ if and only if the linear functional $k \psi_{*}$ defined by the Beltrami differential of $f_{\psi}$ has no degenerate Hamilton sequence, that is, $\psi_{*}$ has no degerenate Hamilton sequence. Therefore, $Q(R)$ is weakly uniformly convex at $\psi$ if and only if $H\left(f_{\psi}\right)<K_{0}\left(f_{\psi}\right)$, by Theorem 1 .

Corollary. Let $\psi \in S(\Delta)$ have a holomorphic extension to a neighbourhood of the closed disk with no zeroes on the unit circle. Then $Q(\Delta)$ is weakly uniformly convex at $\psi$.

Proof. Under the hypothesis, the restriction of $f_{\psi}$ to a neighbourhood of the unit circle is a real analytic diffeomorphism, so $H\left(f_{\psi}\right)=1<K_{0}\left(f_{\psi}\right)$.

Remark. The Corollary applies in particular to $\psi=\frac{n+2}{2 \pi} z^{n} d z^{2} \in S(\Delta)$ and solves Goldberg's Problem 1.

Goldberg [5] shows that $Q(\Delta)$ is nowhere uniformly convex. For any Riemann surface $R$, we have

Theorem 3. (i) If $\operatorname{dim} Q(R)<\infty, Q(R)$ is uniformly convex.

(ii) If $\operatorname{dim} Q(R)=\infty, Q(R)$ is nowhere uniformly convex.

Proof. We have already remarked in $\S 1$ that since $Q(R)$ is strictly convex, it is uniformly convex whenever it is finite dimensional.

Now we suppose that $\operatorname{dim} Q(R)=\infty$. We modify Goldberg's discussion (see [5]). Fix $\psi \in S(R)$ and $\varepsilon>0$. Choose some compact set $K \subset R$ such that $\iint_{K}|\psi(z)| d x d y>1-\varepsilon$. Since $\operatorname{dim} Q(R)=\infty$, there must exist a degenerate sequence $\left(\phi_{n}\right)$ in $S(R)$, that is, $\phi_{n} \rightarrow 0$ locally uniformly in $R$. When $n$ is sufficiently large, $\iint_{S-K}\left|\phi_{n}(z)\right| d x d y>1-\varepsilon$. Therefore,

$$
\begin{aligned}
\left\|\phi_{n} \pm \psi\right\| & =\iint_{K}\left|\phi_{n} \pm \psi\right| d x d y+\iint_{S-K}\left|\phi_{n} \pm \psi\right| d x d y \\
& \geq \iint_{K}|\psi| d x d y-\iint_{K}\left|\phi_{n}\right| d x d y+\iint_{S-K}\left|\phi_{n}\right| d x d y-\iint_{S-K}|\psi| d x d y \geq 2-4 \varepsilon .
\end{aligned}
$$

Thus, $Q(R)$ is not uniformly convex at $\psi \in S(R)$. 


\section{ACKNOWLEDGEMENT}

I wish to thank Prof. Li Zhong for his encouragement and help. I also wish to express my gratitude to the referee for his useful advice and for calling my attention to the result of C.J. Earle and Li Zhong.

\section{REFERENCES}

1. J. Diestel, Geometry of Banach spaces-selected topics, Springer-Verlag, New York, 1975. MR 57:1079

2. C.J. Earle, On holomorphic cross-sections in Teichmüller spaces, Duke Math. J. 36 (1969), 409-416. MR 40:7442

3. C.J. Earle and Li Zhong, Isometrically embedded polydisks in infinite dimensional Teichmüller spaces, to appear.

4. F.P. Gardiner, Teichmüller theory and quadratic differentials, Wiley-Interscience, New York, 1987. MR 88m:32044

5. L.R. Goldberg, On the shape of the unit sphere in $Q(\Delta)$, Proc. Amer. Math. Soc. 118 (1993), 1179-1185. MR 93m:46019

6. A. Harrington and M. Ortel, The dilatation of an extremal quasiconformal mapping, Duke Math. J. 43 (1976), 533-544. MR 54:13074

7. K. Strebel, On quadratic differentials and extremal quasiconformal mappings, Proceedings Int. Congr. Math. Vancouver (1974), 223-227. MR 58:22549

8. K. Strebel, On the existence of extremal Teichmüller mappings, Journal d'Analyse Math. 30 (1976), 464-480. MR 55:12912

9. K. Strebel, Extremal quasiconformal mappings, Resulate Math. 10 (1986), 168-210. MR 88a:30048

Department of Mathematics, Suzhou University, Suzhou 215006, People's Republic OF CHINA 